\title{
Some integral representations and limits for (products of) the parabolic cylinder function
}

\author{
Dirk Veestraeten* \\ University of Amsterdam \\ Amsterdam School of Economics \\ Roetersstraat 11 \\ 1018 WB Amsterdam \\ The Netherlands
}

May 27, 2015

\begin{abstract}
Veestraeten [1] recently derived inverse Laplace transforms for Laplace transforms that contain products of two parabolic cylinder functions by exploiting the link between the parabolic cylinder function and the transition density and distribution functions of the Ornstein-Uhlenbeck process. This paper first uses these results to derive new integral representations for (products of two) parabolic cylinder functions. Second, as the Brownian motion process with drift is a limiting case of the Ornstein-Uhlenbeck process also limits can be calculated for the product of gamma functions and (products of) parabolic cylinder functions. The central results in both cases contain, in stylised form, $D_{v}(x) D_{v}(y)$ and $D_{v}(x) D_{v-1}(y)$ such that the recurrence relation of the parabolic cylinder function straightforwardly allows to obtain integral representations and limits also for countless other combinations in the orders such as $D_{v}(x) D_{v-3}(y)$ and $D_{v+1}(x) D_{v}(y)$.
\end{abstract}

Keywords: Gamma function; integral representations; limits; modified Bessel function; OrnsteinUhlenbeck process; parabolic cylinder function

2010 Mathematics Subject Classification: 33B20; 33C10; 33C15; 44A10; 60J60

*Address correspondence to Dirk Veestraeten, Amsterdam School of Economics, University of Amsterdam, Roetersstraat 11, 1018 WB Amsterdam, The Netherlands, e-mail: d.j.m.veestraeten@uva.nl. 


\section{Introduction}

Veestraeten [1] recently derived inverse Laplace transforms for Laplace transforms that include products of two parabolic cylinder functions of the form $D_{v}(x) D_{v}(y)$ and $D_{v}(x) D_{v-1}(y)$ by exploiting the close link between the parabolic cylinder function and the transition density and distribution functions of the Ornstein-Uhlenbeck process. The recurrence relation for the parabolic cylinder function then was used to obtain inverse transforms also for products of parabolic cylinder functions such as $D_{v}(x) D_{v-3}(y)$ and $D_{v+1}(x) D_{v}(y)$.

This paper first uses these results to calculate integral representations for products of two parabolic cylinder functions with differing arguments and that have the same or different order. The result for the product in which the parabolic cylinder functions share the same order corresponds with the integral representations that were obtained in Malyshev [2] and Glasser [3]. Our methodology also allows to derive multiple representations for products that have the same combination of orders in the two parabolic cylinder functions. Furthermore, for orders -1 and $-1 / 2$, the parabolic cylinder function simplifies into the complementary error function, $\operatorname{erfc}(x)$, and the modified Bessel function of order $1 / 4, K_{1 / 4}(x)$, respectively, such that also integral representations can be obtained for $\operatorname{erfc}(x) \operatorname{erfc}(y), K_{1 / 4}(x) K_{1 / 4}(y), K_{1 / 4}(x), K_{1 / 4}(x) D_{-3 / 2}(y)$, etc.

Second, the Brownian motion process with drift can be obtained from the Ornstein-Uhlenbeck process by letting the mean-reversion parameter $\beta$ in the latter process approach 0 (see Cox and Miller [4). This property enables the derivation of limits for expressions that contain gamma functions and (products of) parabolic cylinder functions in which the order in the latter functions approaches negative infinity. These limits do not yield asymptotic expansions for parabolic cylinder functions (see Olver [5]), but evaluate into exponential functions that are independent of $\beta$ akin to the results for other special functions that are obtained in Brychkov [6].

This paper illustrates the basic relations and presents some examples. However, the recurrence relation of the parabolic cylinder function can be used to extend results to various other orders in the (product of) parabolic cylinder functions. The remainder of the paper is organised as follows. Section 2 lists the results of Veestraeten [1] that will be used throughout the paper and subsequently derives integral representations for (products of two) parabolic cylinder functions. Section 3 discusses the relation between the (Laplace transforms of the) Ornstein-Uhlenbeck process and the Brownian motion process and obtains several limits that include gamma functions and (products of) parabolic cylinder functions.

\section{Integral representations for (products of) parabolic cylinder functions and related special functions}

The below integral representations and limits are calculated on the basis of six inverse Laplace transforms that were derived in Veestraeten [1. For ease of reference, these results are reproduced 
in Table 1.

Table 1: around here.

Using $v=-\frac{s+c}{\beta}$ in the first entry of Table 1 gives the following integral representation for the product of two parabolic cylinder functions of the same order but with differing arguments

$$
\begin{aligned}
& D_{v}(x) D_{v}(y)=\frac{\exp \left(\frac{y^{2}-x^{2}}{4}\right)}{\Gamma(-v)} \int_{0}^{+\infty} \frac{\beta \exp [\beta v t]}{\sqrt{1-\exp (-2 \beta t)}} \exp \left(-\frac{(y+x \exp (-\beta t))^{2}}{2(1-\exp (-2 \beta t))}\right) d t \\
& {[\operatorname{Re} v<0, \beta>0, x+y \geqslant 0] .}
\end{aligned}
$$

The substitution $u=1-\exp (-2 \beta t)$ allows to write the latter result more compactly

$$
\begin{aligned}
& D_{v}(x) D_{v}(y)=\frac{\exp \left(\frac{y^{2}-x^{2}}{4}\right)}{2 \Gamma(-v)} \int_{0}^{1} \frac{1}{(1-u)^{1+\frac{v}{2}} \sqrt{u}} \exp \left(-\frac{(y+x \sqrt{1-u})^{2}}{2 u}\right) d u \\
& {[\operatorname{Re} v<0, x+y \geqslant 0]}
\end{aligned}
$$

which, together with the other central integral representations, is listed in Table 2 .

\section{Table 2: around here.}

Glasser [3] recently derived the following integral representation for $D_{-v}(x) D_{-v}(-y)$ under $\operatorname{Re} v>$ 0 and $x>y>0$

$$
\begin{aligned}
& D_{-v}(x) D_{-v}(-y)= \\
& \quad \frac{\exp \left(-\frac{1}{4}\left(x^{2}+y^{2}\right)\right)}{2 \Gamma(v)} \int_{0}^{\infty} t^{v / 2-1}(t+1)^{-(v+1) / 2} \exp \left(-\frac{1}{2}\left(x^{2}+y^{2}\right) t+x y \sqrt{t(t+1)}\right) d t .
\end{aligned}
$$

Using the substitutions $u=\frac{1}{q}$ and subsequently $q=t+1$ within the integral (2.2) reveals that both expressions are identical. Note that the requirement in Glasser [3] of the arguments having opposite sign is too restrictive.

Applying the steps that were used for the integral representation (2.2) to the second entry in Table 1 produces

$$
\begin{aligned}
& D_{v}(x) D_{v-1}(y)=\frac{\sqrt{\pi} \exp \left(\frac{y^{2}-x^{2}}{4}\right)}{2^{\frac{3}{2}} \Gamma(-v)} \int_{0}^{1} \frac{1}{(1-u)^{1+\frac{v}{2}}} \operatorname{erfc}\left(\frac{y+x \sqrt{1-u}}{\sqrt{2 u}}\right) d u \\
& {[\operatorname{Re} v<0, x+y \geqslant 0]}
\end{aligned}
$$

where $\operatorname{erfc}(z)$ is the complementary error function, see Abramowitz and Stegun [7]. Setting $c=0$ in the third entry in Table 1 yields a further representation for $D_{v}(x) D_{v-1}(y)$ that instead relies 
on the exponential function

$$
\begin{aligned}
& D_{v}(x) D_{v-1}(y)= \\
& -\frac{\exp \left(\frac{y^{2}-x^{2}}{4}\right)}{2 v \Gamma(-v)} \int_{0}^{1} \frac{x+y \sqrt{1-u}}{(1-u)^{\frac{1+v}{2}} u^{\frac{3}{2}}} \exp \left(-\frac{(y+x \sqrt{1-u})^{2}}{2 u}\right) d u-\left.\frac{\sqrt{\pi}}{v \sqrt{2} \Gamma(-v)}\right|_{x+y=0} \\
& \quad[\operatorname{Re} v<0, x+y \geqslant 0] .
\end{aligned}
$$

Note that the term $\frac{\sqrt{\pi}}{v \sqrt{2} \Gamma(-v)}$ is to be subtracted when the sum of the arguments equals 0 for which the notation $\left.\frac{\sqrt{\pi}}{v \sqrt{2} \Gamma(-v)}\right|_{x+y=0}$ is employed. This additional term originated from the use of the differentiation property of the Laplace transform in deriving the third entry in Table 1, see Veestraeten [1] for more detail.

The inverse Laplace transforms in entries $4-6$ in Table 1 specify the following three integral representations for $D_{v}(x) D_{v-2}(y)$

$$
\begin{gathered}
D_{v}(x) D_{v-2}(y)=\frac{\exp \left(\frac{y^{2}-x^{2}}{4}\right)}{2 \Gamma(-v)} \int_{0}^{1} \frac{1}{(1-u)^{1+\frac{v}{2}}}\left\{\sqrt{u} \exp \left(-\frac{(y+x \sqrt{1-u})^{2}}{2 u}\right)\right. \\
\left.-\sqrt{\frac{\pi}{2}}(x \sqrt{1-u}+y) \operatorname{erfc}\left(\frac{y+x \sqrt{1-u}}{\sqrt{2 u}}\right)\right\} d u \\
{[\operatorname{Re} v<0, x+y \geqslant 0],}
\end{gathered}
$$

and

$$
\begin{aligned}
& D_{v}(x) D_{v-2}(y)=-\frac{\exp \left(\frac{y^{2}-x^{2}}{4}\right)}{2 v \Gamma(-v)} \int_{0}^{1} \frac{1}{(1-u)^{1+\frac{v}{2}}}\left\{\frac{1-u}{\sqrt{u}} \exp \left(-\frac{(y+x \sqrt{1-u})^{2}}{2 u}\right)\right. \\
& \left.+x \sqrt{\frac{\pi}{2}(1-u)} \operatorname{erfc}\left(\frac{y+x \sqrt{1-u}}{\sqrt{2 u}}\right)\right\} d u \\
& {[\operatorname{Re} v<0, x+y \geqslant 0],}
\end{aligned}
$$

and

$$
\begin{aligned}
& D_{v}(x) D_{v-2}(y)=\frac{\exp \left(\frac{y^{2}-x^{2}}{4}\right)}{2(1-v) \Gamma(-v)} \int_{0}^{1} \frac{1}{(1-u)^{1+\frac{v}{2}}}\left\{\frac{1}{\sqrt{u}} \exp \left(-\frac{(y+x \sqrt{1-u})^{2}}{2 u}\right)\right. \\
& \left.-y \sqrt{\frac{\pi}{2}} \operatorname{erfc}\left(\frac{y+x \sqrt{1-u}}{\sqrt{2 u}}\right)\right\} d u \\
& {[\operatorname{Re} v<0, x+y \geqslant 0] .}
\end{aligned}
$$

The integral (2.5) is a linear combination of integrals (2.6) and (2.7) with weights $v$ and $(1-v)$, respectively. The integral representations (2.6) and (2.7) mainly differ in the definition of the weights of the exponential function and the complementary error function with the weight of the latter function depending on either $x$ or $y$, respectively. An additional, more compact integral representation for $D_{v}(x) D_{v-2}(y)$ can be obtained via the recurrence relation

$$
D_{v+1}(z)-z D_{v}(z)+v D_{v-1}(z)=0,
$$


see Equation (8.2.14) in Erdélyi et al. 8]. Relation (2.8) yields

$$
\begin{aligned}
& D_{v-2}(y)=\frac{y}{v-1} D_{v-1}(y)-\frac{1}{v-1} D_{v}(y) \\
& D_{v}(x) D_{v-2}(y)=\frac{y}{v-1} D_{v}(x) D_{v-1}(y)-\frac{1}{v-1} D_{v}(x) D_{v}(y) .
\end{aligned}
$$

Plugging the integrals (2.4) and (2.2) into the latter expression gives

$$
\begin{aligned}
& D_{v}(x) D_{v-2}(y)= \\
& \frac{\exp \left(\frac{y^{2}-x^{2}}{4}\right)}{2 v(1-v) \Gamma(-v)} \int_{0}^{1} \frac{y \sqrt{1-u}(x+y \sqrt{1-u})+v u}{(1-u)^{1+\frac{v}{2}} u^{\frac{3}{2}}} \exp \left(-\frac{(y+x \sqrt{1-u})^{2}}{2 u}\right) d u \\
& -\left.\frac{y \sqrt{\pi}}{v(v-1) \sqrt{2} \Gamma(-v)}\right|_{x+y=0} \\
& \quad[\operatorname{Re} v<0, x+y \geqslant 0] .
\end{aligned}
$$

Likewise, the recurrence relation $D_{v}(x) D_{v+1}(y)=y D_{v}(x) D_{v}(y)-v D_{v}(x) D_{v-1}(y)$ together with integrals (2.2) and (2.4) gives

$$
\begin{aligned}
& D_{v}(x) D_{v+1}(y)=\frac{\exp \left(\frac{y^{2}-x^{2}}{4}\right)}{2 \Gamma(-v)} \int_{0}^{1} \frac{y u+\sqrt{1-u}(x+y \sqrt{1-u})}{(1-u)^{1+\frac{v}{2}} u^{\frac{3}{2}}} \exp \left(-\frac{(y+x \sqrt{1-u})^{2}}{2 u}\right) d u \\
& +\left.\frac{\sqrt{\pi}}{\sqrt{2} \Gamma(-v)}\right|_{x+y=0} \\
& \quad[\operatorname{Re} v<0, x+y \geqslant 0] .
\end{aligned}
$$

Repeating the above steps also produces (multiple) integral representations for products of parabolic cylinder functions that have more distant orders such as $D_{v}(x) D_{v+5}(y)$ and $D_{v}(x) D_{v-5}(y)$.

The above integral representations are valid for $x+y \geqslant 0$ such that they can easily be modified to allow for specific values in the arguments. For instance, applying $\beta=1$ and $y=-x$ to Equation (2.1) gives

$$
D_{v}(x) D_{v}(-x)=\frac{1}{\Gamma(-v)} \int_{0}^{+\infty} \frac{\exp [v t]}{\sqrt{1-\exp (-2 t)}} \exp \left(-\frac{x^{2}(1-\exp (-t))^{2}}{2(1-\exp (-2 t))}\right) d t
$$

$[\operatorname{Re} v<0]$,

which now holds for all real arguments. The latter integral, given the identity $\tanh \frac{t}{2}=(\cosh t-1) / \sinh t$, can be rewritten as

$$
\begin{aligned}
& D_{v}(x) D_{v}(-x)=2^{-1 / 2} \Gamma^{-1}(-v) \int_{0}^{+\infty} \exp \left(\left(v+\frac{1}{2}\right) t-\frac{x^{2}}{2} \tanh \frac{t}{2}\right) \frac{d t}{\sqrt{\sinh t}} \\
& \quad[\operatorname{Re} v<0],
\end{aligned}
$$

which is the result in Equation (6) of Malyshev [2]. 
The above expressions also generate (multiple) representations for single parabolic cylinder functions. This is illustrated using Equation (2.3) for $y=0$ together with the identity

$$
D_{v}(0)=\frac{2^{v / 2} \sqrt{\pi}}{\Gamma\left(\frac{1-v}{2}\right)},
$$

see Magnus, Oberhettinger and Soni [9], where $\Gamma(v)$ denotes the gamma function. Further simplifications via the recurrence and the duplication formulas for the gamma function, i.e. $\Gamma(v+1)=$ $v \Gamma(v)$ and $\Gamma(2 v)=\frac{1}{\sqrt{2 \pi}} 2^{2 v-\frac{1}{2}} \Gamma(v) \Gamma\left(v+\frac{1}{2}\right)$ in Equations (6.1.15) and (6.1.18) in Abramowitz and Stegun [7, then give

$$
\begin{aligned}
& D_{v}(x)=-\frac{v \sqrt{\pi} 2^{\frac{1}{2} v-1} \exp \left(\frac{-x^{2}}{4}\right)}{\Gamma\left(\frac{1}{2}-\frac{1}{2} v\right)} \int_{0}^{1} \frac{1}{(1-u)^{1+\frac{v}{2}}} \operatorname{erfc}\left(\frac{x \sqrt{1-u}}{\sqrt{2 u}}\right) d u \\
& {[\operatorname{Re} v<0, x \geqslant 0] .}
\end{aligned}
$$

The latter integral is expressed in terms of the complementary error function and has not been documented in, for instance, Erdélyi et al. 8] and Gradshteyn and Ryzhik [10].

Choosing specific values for the order creates integral representations for (products of) a number of other special functions. For instance, the parabolic cylinder function simplifies into the complementary error function for $v=-1$ via

$$
D_{-1}(z)=\sqrt{\frac{\pi}{2}} \exp \left(\frac{z^{2}}{4}\right) \operatorname{erfc}\left(\frac{z}{\sqrt{2}}\right)
$$

see Equation (9.254.1) in Gradshteyn and Ryzhik [10]. Using $\beta=s+c$ within the first entry of Table 1, applying the substitution $u=1-\exp (-2 \beta t)$ and rescaling $x$ and $y$ gives the following compact integral representation for the product of two complementary error functions

$$
\begin{aligned}
& \operatorname{erfc}(x) \operatorname{erfc}(y)=\frac{\exp \left(-x^{2}\right)}{\pi} \int_{0}^{1} \frac{1}{\sqrt{u(1-u)}} \exp \left(-\frac{(y+x \sqrt{1-u})^{2}}{u}\right) d u \\
& {[x+y \geqslant 0]}
\end{aligned}
$$

which has not been documented in, for instance, the overview of $\mathrm{Ng}$ and Geller [11].

The parabolic cylinder function with order $v=-\frac{1}{2}$ is related to the modified Bessel function with order $\frac{1}{4}$ via

$$
D_{-\frac{1}{2}}(z)=\frac{\sqrt{z}}{\sqrt{2 \pi}} K_{\frac{1}{4}}\left(\frac{1}{4} z^{2}\right)
$$

see Magnus, Oberhettinger and Soni [9]. Setting $y=0$ in the first entry of Table 1 and using $\frac{1}{2} \beta=s+c$ yields

$$
\begin{aligned}
& K_{1 / 4}(x)=\frac{\sqrt{\pi}}{(2 x)^{\frac{1}{4}} \Gamma\left[\frac{1}{4}\right]} \int_{0}^{1} \frac{1}{(1-u)^{\frac{3}{4}} \sqrt{u}} \exp \left(-\frac{x(2-u)}{u}\right) d u \\
& {[x>0],}
\end{aligned}
$$


and an alternative representation in terms of the complementary error function can be obtained from entry 2 in Table 1.

The above results also generate integral representations for combinations of the aforementioned three special functions. For instance, entry 2 in Table 1 with $\frac{1}{2} \beta=s+c$ gives

$$
\begin{aligned}
& K_{\frac{1}{4}}(x) D_{-\frac{3}{2}}(y)=\frac{\sqrt{\pi} \exp \left(\frac{y^{2}-4 x}{4}\right)}{2^{\frac{3}{2}} x^{\frac{1}{4}}} \int_{0}^{1} \frac{1}{(1-u)^{\frac{3}{4}}} \operatorname{erfc}\left(\frac{y+2 \sqrt{x(1-u)}}{\sqrt{2 u}}\right) d u \\
& {[x>0,2 \sqrt{x}+y \geqslant 0] .}
\end{aligned}
$$

\section{Limits containing (products of) parabolic cylinder functions}

The Brownian motion process with drift is a limiting case of the Ornstein-Uhlenbeck process (see Cox and Miller [4]) and this property then also applies to the Laplace transforms for the transition density and distribution functions of both processes. This section first lists the Laplace transforms for the Ornstein-Uhlenbeck process that were derived in Veestraeten [1] and subsequently obtains the transforms for the Brownian motion process with drift.

Let $W=\left\{W_{t}, t \geqslant 0\right\}$ be an Ornstein-Uhlenbeck process with initial value $W_{0}=w_{0}$. The dynamics of this stochastic process are

$$
d W_{t}=\left(\alpha-\beta W_{t}\right) d t+\sigma d Z_{t}
$$

with $\beta>0$ and $t>0$. The instantaneous variance is $\sigma^{2}$, with $\sigma>0$, and $d Z_{t}$ denotes the increment of a Wiener process. The process (3.1) is mean-reverting since the positive value of $\beta$ ensures that the stochastic variable reverts to its long-term mean $\frac{\alpha}{\beta}$.

The transition probability distribution function - in short, the transition distribution - is defined as $P\left(w, t \mid w_{0}\right)=\operatorname{Pr}\left\{W(t) \leqslant w \mid W(0)=w_{0}\right\}$ and the transition probability density function - in short, the transition density - is given by $p\left(w, t \mid w_{0}\right)=\frac{\partial}{\partial w} P\left(w, t \mid w_{0}\right)$. The Laplace transform of the transition density of the Ornstein-Uhlenbeck process, $\bar{p}\left(w, s \mid w_{0}\right)$, was derived in Equation (3.3) in Veestraeten [1] as

$$
\bar{p}\left(w, s \mid w_{0}\right)=\left\{\begin{aligned}
\frac{\Gamma(s / \beta)}{\sigma \sqrt{\pi \beta}} \exp \left(\frac{\left(\beta w_{0}-\alpha\right)^{2}}{2 \sigma^{2} \beta}-\frac{(\beta w-\alpha)^{2}}{2 \sigma^{2} \beta}\right) \\
\quad \times D_{-s / \beta}\left(\frac{\sqrt{2}(\beta w-\alpha)}{\sigma \sqrt{\beta}}\right) D_{-s / \beta}\left(-\frac{\sqrt{2}\left(\beta w_{0}-\alpha\right)}{\sigma \sqrt{\beta}}\right) \text { for }-\infty \leqslant w_{0} \leqslant w \\
\frac{\Gamma(s / \beta)}{\sigma \sqrt{\pi \beta}} \exp \left(\frac{\left(\beta w_{0}-\alpha\right)^{2}}{2 \sigma^{2} \beta}-\frac{(\beta w-\alpha)^{2}}{2 \sigma^{2} \beta}\right) \\
\quad \times D_{-s / \beta}\left(-\frac{\sqrt{2}(\beta w-\alpha)}{\sigma \sqrt{\beta}}\right) D_{-s / \beta}\left(\frac{\sqrt{2}\left(\beta w_{0}-\alpha\right)}{\sigma \sqrt{\beta}}\right) \text { for } w \leqslant w_{0} \leqslant+\infty
\end{aligned}\right.
$$


and the Laplace transform for the transition distribution, $\bar{P}\left(w_{1}, s \mid w_{0}\right)$ with $w_{1} \geqslant w_{0}$, was specified in Equation (3.5) as

$$
\begin{aligned}
\bar{P}\left(w_{1}, s \mid w_{0}\right) & =\frac{1}{s}-\frac{\Gamma(s / \beta)}{\beta \sqrt{2 \pi}} \exp \left(\frac{\left(\beta w_{0}-\alpha\right)^{2}}{2 \sigma^{2} \beta}-\frac{\left(\beta w_{1}-\alpha\right)^{2}}{2 \sigma^{2} \beta}\right) \\
& \times D_{-s / \beta}\left(-\frac{\sqrt{2}\left(\beta w_{0}-\alpha\right)}{\sigma \sqrt{\beta}}\right) D_{-1-s / \beta}\left(\frac{\sqrt{2}\left(\beta w_{1}-\alpha\right)}{\sigma \sqrt{\beta}}\right) \text { for } w_{1} \geqslant w_{0} .
\end{aligned}
$$

The dynamic process for Brownian motion with drift coefficient $\alpha$ is given by

$$
d W_{t}=\alpha d t+\sigma d Z_{t}
$$

with $t>0$ and thus can be seen as the limit of the Ornstein-Uhlenbeck process (3.1) for $\beta$ approaching 0. The transition density of the Brownian motion, $p^{B M}\left(w, t \mid w_{0}\right)$, satisfies the Kolmogorov forward equation (see Cox and Miller [4] and Risken [12])

$$
\frac{1}{2} \sigma^{2} \frac{\partial^{2} p^{B M}\left(w, t \mid w_{0}\right)}{\partial w^{2}}-\alpha \frac{\partial p^{B M}\left(w, t \mid w_{0}\right)}{\partial w}=\frac{\partial p^{B M}\left(w, t \mid w_{0}\right)}{\partial t} .
$$

The initial condition is

$$
p^{B M}\left(w, t \mid w_{0}\right)=\delta\left(w-w_{0}\right) \quad \text { for } t=0,
$$

where $\delta(\cdot)$ is the Dirac delta function that implies that all initial probability mass is located on the initial state.

The Laplace transform of the transition density, $\bar{p}^{B M}\left(w, s \mid w_{0}\right)$, is defined as

$$
\bar{p}^{B M}\left(w, s \mid w_{0}\right)=\int_{0}^{+\infty} \exp (-s t) p^{B M}\left(w, t \mid w_{0}\right) d t,
$$

where Re $s>0$. The Kolmogorov forward equation then simplifies into

$$
\frac{1}{2} \sigma^{2} \frac{d^{2} \bar{p}^{B M}\left(w, s \mid w_{0}\right)}{d w^{2}}-\alpha \frac{d \bar{p}^{B M}\left(w, s \mid w_{0}\right)}{d w}-s \bar{p}^{B M}\left(w, s \mid w_{0}\right)=-\delta\left(w-w_{0}\right)
$$

and its solution is

$$
\bar{p}^{B M}\left(w, s \mid w_{0}\right)=\left\{\begin{array}{l}
\frac{1}{\sqrt{\alpha^{2}+2 s \sigma^{2}}} \exp \left(\frac{-\alpha+\sqrt{\alpha^{2}+2 s \sigma^{2}}}{\sigma^{2}}\left(w_{0}-w\right)\right) \text { for }-\infty \leqslant w_{0} \leqslant w \\
\frac{1}{\sqrt{\alpha^{2}+2 s \sigma^{2}}} \exp \left(\frac{-\alpha-\sqrt{\alpha^{2}+2 s \sigma^{2}}}{\sigma^{2}}\left(w_{0}-w\right)\right) \text { for } w \leqslant w_{0} \leqslant+\infty .
\end{array}\right.
$$

The transition distribution, $P^{B M}\left(w_{1}, t \mid w_{0}\right)$, specifies the cumulative probability mass that is located below $w_{1}$. Its Laplace transform can be obtained by integrating the Laplace transform of the transition density

$$
\bar{P}^{B M}\left(w_{1}, s \mid w_{0}\right)=\int_{-\infty}^{w_{1}} \bar{p}^{B M}\left(w, s \mid w_{0}\right) d w
$$


see Veestraeten [1]. Then, $\bar{P}^{B M}\left(w_{1}, s \mid w_{0}\right)$ is given by

$$
\begin{aligned}
& \bar{P}^{B M}\left(w_{1}, s \mid w_{0}\right)=\frac{1}{s}-\frac{\sigma^{2}}{\sqrt{\alpha^{2}+2 s \sigma^{2}}\left(\sqrt{\alpha^{2}+2 s \sigma^{2}}-\alpha\right)} \exp \left(\frac{-\alpha+\sqrt{\alpha^{2}+2 s \sigma^{2}}}{\sigma^{2}}\left(w_{0}-w_{1}\right)\right) \\
& \quad \text { for } w_{1} \geqslant x_{0} .
\end{aligned}
$$

The Laplace transforms for the transition densities (3.2) and (3.4) are related as follows

$$
\begin{aligned}
\lim _{\beta \rightarrow 0} & {\left[\frac{\Gamma(s / \beta)}{\sigma \sqrt{\pi \beta}} D_{-s / \beta}\left(\frac{\sqrt{2}(\beta w-\alpha)}{\sigma \sqrt{\beta}}\right) D_{-s / \beta}\left(-\frac{\sqrt{2}\left(\beta w_{0}-\alpha\right)}{\sigma \sqrt{\beta}}\right)\right] } \\
& =\frac{1}{\sqrt{\alpha^{2}+2 s \sigma^{2}}} \exp \left(\frac{\sqrt{\alpha^{2}+2 s \sigma^{2}}}{\sigma^{2}}\left(w_{0}-w\right)\right) \quad \text { for }-\infty \leqslant w_{0} \leqslant w .
\end{aligned}
$$

Setting $\sigma, w$ and $w_{0}$ at $\sqrt{2}, x$ and $y$, respectively, gives

$$
\begin{aligned}
\lim _{\beta \rightarrow 0} & {\left[\frac{\Gamma(s / \beta)}{\sqrt{\beta}} D_{-s / \beta}\left(x \sqrt{\beta}-\frac{\alpha}{\sqrt{\beta}}\right) D_{-s / \beta}\left(y \sqrt{\beta}+\frac{\alpha}{\sqrt{\beta}}\right)\right]=} \\
& \frac{\sqrt{2 \pi}}{\sqrt{\alpha^{2}+4 s}} \exp \left(-\frac{\sqrt{\alpha^{2}+4 s}}{2}(x+y)\right)
\end{aligned}
$$

$[\operatorname{Re} s>0]$,

which, together with the other basic relations that follow, is also listed in Table 3. Again, $x$ and $y$ are real but, in contrast to the above integral representations, no condition is required as to their sum. Note that the limit (3.7) also arises when instead evaluating the Laplace transforms for the domain $w \leqslant w_{0} \leqslant+\infty$.

\section{Table 3: around here.}

The orders of the parabolic cylinder functions in the limits in this paper thus approach negative infinity with arguments that vanish $(\alpha=0)$ or that approach $+\infty$ or $-\infty$ depending on the sign of $\alpha$.

The Laplace transforms for the transition distributions (3.3) and (3.5), using the above notation, yield

$$
\begin{gathered}
\lim _{\beta \rightarrow 0}\left[\frac{\Gamma(s / \beta)}{\beta} D_{-s / \beta}\left(y \sqrt{\beta}+\frac{\alpha}{\sqrt{\beta}}\right) D_{-1-s / \beta}\left(x \sqrt{\beta}-\frac{\alpha}{\sqrt{\beta}}\right)\right]= \\
\frac{2 \sqrt{2 \pi}}{\sqrt{\alpha^{2}+4 s}\left(\sqrt{\alpha^{2}+4 s}-\alpha\right)} \exp \left(-\frac{\sqrt{\alpha^{2}+4 s}}{2}(x+y)\right) \\
{[\operatorname{Re} s>0] .}
\end{gathered}
$$

The recurrence relation (2.8) allows to expand the above results to other orders. For instance, using $v=-1-s / \beta$ in the recurrence relation, applying it to the second parabolic cylinder function in 
the product and keeping the order of the first parabolic cylinder function at $-s / \beta$ gives

$$
\begin{aligned}
\lim _{\beta \rightarrow 0}[ & \left.\frac{(s+\beta) \Gamma(s / \beta)}{\beta^{\frac{3}{2}}} D_{-s / \beta}\left(y \sqrt{\beta}+\frac{\alpha}{\sqrt{\beta}}\right) D_{-2-s / \beta}\left(x \sqrt{\beta}-\frac{\alpha}{\sqrt{\beta}}\right)\right]= \\
& \lim _{\beta \rightarrow 0}\left[\frac{-(x \beta-\alpha) \Gamma(s / \beta)}{\beta} D_{-s / \beta}\left(y \sqrt{\beta}+\frac{\alpha}{\sqrt{\beta}}\right) D_{-1-s / \beta}\left(x \sqrt{\beta}-\frac{\alpha}{\sqrt{\beta}}\right)\right] \\
& +\lim _{\beta \rightarrow 0}\left[\frac{\Gamma(s / \beta)}{\sqrt{\beta}} D_{-s / \beta}\left(y \sqrt{\beta}+\frac{\alpha}{\sqrt{\beta}}\right) D_{-s / \beta}\left(x \sqrt{\beta}-\frac{\alpha}{\sqrt{\beta}}\right)\right] .
\end{aligned}
$$

The first term on the right-hand side equals $\frac{2 \alpha \sqrt{2 \pi}}{\sqrt{\alpha^{2}+4 s}\left(\sqrt{\alpha^{2}+4 s}-\alpha\right)} \exp \left(-\frac{\sqrt{\alpha^{2}+4 s}}{2}(x+y)\right)$ as can be inferred from Equation (3.8) and the second right-hand side term is given in Equation (3.7) such that

$$
\begin{aligned}
\lim _{\beta \rightarrow 0}\left[\frac{(s+\beta) \Gamma(s / \beta)}{\beta^{\frac{3}{2}}} D_{-s / \beta}\left(y \sqrt{\beta}+\frac{\alpha}{\sqrt{\beta}}\right) D_{-2-s / \beta}\left(x \sqrt{\beta}-\frac{\alpha}{\sqrt{\beta}}\right)\right]= \\
\frac{\sqrt{2 \pi}\left(\alpha+\sqrt{\alpha^{2}+4 s}\right)}{\sqrt{\alpha^{2}+4 s}\left(\sqrt{\alpha^{2}+4 s}-\alpha\right)} \exp \left(-\frac{\sqrt{\alpha^{2}+4 s}}{2}(x+y)\right) \\
{[\operatorname{Re} s>0] . }
\end{aligned}
$$

Limits for other orders can straightforwardly be obtained by applying the recurrence relation in Equation (2.8) to the above limits.

The limiting case for a single parabolic cylinder function can directly be evaluated via the limits (3.7)-(3.9) and the identity (2.11). However, this is not attractive as it requires $\alpha=0$ and thus obscures the role of $\alpha$. Instead, the limiting case for a single parabolic cylinder function within the limit (3.6) is obtained by using $w_{0}=\frac{\alpha}{\beta}$. The right-hand side then amounts to $\frac{1}{\sqrt{\alpha^{2}+2 s \sigma^{2}}} \exp \left(\frac{\sqrt{\alpha^{2}+2 s \sigma^{2}}}{\sigma^{2}}\left(\frac{\alpha}{\beta}-w\right)\right)$, which is 0 for $\alpha<0,+\infty$ for $\alpha>0$ and $\frac{1}{\sqrt{2 s \sigma^{2}}} \exp \left(-\frac{\sqrt{2 s \sigma^{2}}}{\sigma^{2}} w\right)$ for $\alpha=0$. Using $\sigma=\sqrt{2}, x=w$ and the identity (2.11) gives

$$
\begin{gathered}
\lim _{\beta \rightarrow 0}\left[\frac{2^{s /(2 \beta)} \Gamma(s /(2 \beta))}{\sqrt{\beta}} D_{-s / \beta}\left(x \sqrt{\beta}-\frac{\alpha}{\sqrt{\beta}}\right)\right]= \begin{cases}0 & \alpha<0 \\
\sqrt{\frac{2 \pi}{s}} \exp (-\sqrt{s} x) & \alpha=0 \\
+\infty & \alpha>0\end{cases} \\
{[\operatorname{Re} s>0] .}
\end{gathered}
$$

The following two limits are obtained from the relation for the transition distribution

$$
\lim _{\beta \rightarrow 0}\left[2^{s /(2 \beta)} \Gamma((s+\beta) /(2 \beta)) D_{-s / \beta}\left(x \sqrt{\beta}-\frac{\alpha}{\sqrt{\beta}}\right)\right]= \begin{cases}0 & \alpha<0 \\ \sqrt{\pi} \exp (-\sqrt{s} x) & \alpha=0 \\ +\infty & \alpha>0\end{cases}
$$$$
[\operatorname{Re} s>0]
$$ 
and

$$
\lim _{\beta \rightarrow 0}\left[\frac{2^{s /(2 \beta)} \Gamma(s /(2 \beta))}{\beta} D_{-1-s / \beta}\left(x \sqrt{\beta}-\frac{\alpha}{\sqrt{\beta}}\right)\right]= \begin{cases}0 & \alpha<0 \\ \frac{\sqrt{2 \pi}}{s} \exp (-\sqrt{s} x) & \alpha=0 \\ +\infty & \alpha>0\end{cases}
$$

$[\operatorname{Re} s>0]$.

Applying the recurrence relation (2.8) to the limits (3.12) and (3.10) yields

$$
\lim _{\beta \rightarrow 0}\left[\frac{2^{s /(2 \beta)}(s+\beta) \Gamma(s /(2 \beta))}{\beta^{\frac{3}{2}}} D_{-2-s / \beta}\left(x \sqrt{\beta}-\frac{\alpha}{\sqrt{\beta}}\right)\right]= \begin{cases}0 & \alpha<0 \\ \sqrt{\frac{2 \pi}{s}} \exp (-\sqrt{s} x) & \alpha=0 \\ +\infty & \alpha>0\end{cases}
$$

$[\operatorname{Re} s>0]$.

Note that setting the arguments in both parabolic cylinder functions at 0 within the above relations produces the following limit for the ratio of gamma functions

$$
\begin{gathered}
\lim _{\beta \rightarrow 0}\left[\frac{\Gamma(s /(2 \beta))}{\sqrt{\beta} \Gamma((s+\beta) /(2 \beta))}\right]=\sqrt{\frac{2}{s}} \\
{[\operatorname{Re} s>0] .}
\end{gathered}
$$

This result actually is a special case of the limit (1.18.5) in Erdélyi et al. [13]

$$
\lim _{|z| \rightarrow+\infty}[\exp (-a \log (z)) \Gamma(z+a) / \Gamma(z)]=1,
$$

which in our notation can be written as $\lim _{\beta \rightarrow 0}[\exp (-a \log (1 / \beta)) \Gamma(1 / \beta+a) / \Gamma(1 / \beta)]=1$. Using $a=\frac{1}{2}$ in the latter limit and choosing $s=2$ in the limit (3.14) produces identical specifications.

The above results can also be used to evaluate limits for products of four parabolic cylinder functions and various ratios. For instance, dividing the limit (3.7) by the limit (3.8) gives

$$
\begin{gathered}
\lim _{\beta \rightarrow 0}\left[\sqrt{\beta} D_{-s / \beta}\left(x \sqrt{\beta}-\frac{\alpha}{\sqrt{\beta}}\right) / D_{-1-s / \beta}\left(x \sqrt{\beta}-\frac{\alpha}{\sqrt{\beta}}\right)\right]=\frac{\sqrt{\alpha^{2}+4 s}-\alpha}{2} \\
{[\operatorname{Re} s>0],}
\end{gathered}
$$

which offers neat specialisations. For instance, using $x=0$ and $\alpha=s=1$ gives a limit in which both the order as well as the argument approach negative infinity

$$
\lim _{\beta \rightarrow 0}\left[\sqrt{\beta} D_{-1 / \beta}\left(-\frac{1}{\sqrt{\beta}}\right) / D_{-1-1 / \beta}\left(-\frac{1}{\sqrt{\beta}}\right)\right]=\frac{\sqrt{5}-1}{2} .
$$

Using $s=4$ and $x=1$ and letting the arguments approach 0 via $\alpha=0$ yields

$$
\lim _{\beta \rightarrow 0}\left[\left(\sqrt{\beta} D_{-4 / \beta}(\sqrt{\beta})\right) / D_{-1-4 / \beta}(\sqrt{\beta})\right]=2 .
$$




\section{References}

[1] Veestraeten D. On the inverse transform of Laplace transforms that contain (products of) the parabolic cylinder function. E-print arXiv:1505.05603v1.

[2] Malyshev C. A Nicholson-Type Integral for the Product of Two Parabolic Cylinder Functions $D_{v}(x) D_{v}(-x)$ at $\mathfrak{R} v<0$. Integr. Transf. Spec. F. 2003;14:139-148.

[3] Glasser ML. An integral representation for the product of two parabolic cylinder functions having unrelated arguments. E-print arXiv:1502.00102 v1.

[4] Cox DR, Miller HD. The Theory of Stochastic Processes. London: Chapman \& Hall; 1972.

[5] Olver FWJ. Uniform Asymptotic Expansions for Weber Parabolic Cylinder Functions of Large Orders. J. Research NBS. 1959;63B:131-169.

[6] Brychkov YA. Handbook of Special Functions. Derivatives, Integrals, Series and Other Formulas. Boca Raton (FL): Chapman \& Hall/CRC Press; 2008.

[7] Abramowitz M, Stegun IA. Handbook of Mathematical Functions with Formulas, Graphs, and Mathematical Tables. New York (NY): Dover Publications; 1972.

[8] Erdélyi A, Magnus W, Oberhettinger F, Tricomi FG. Higher Transcendental Functions. Vol. 2. New York (NY): McGraw-Hill; 1953.

[9] Magnus W, Oberhettinger F, Soni RP. Formulas and Theorems for the Special Functions of Mathematical Physics. 3rd ed. Berlin: Springer; 1966.

[10] Gradshteyn IS, Ryzhik IM. Table of Integrals, Series, and Products. Editors: Jeffrey A, Zwillinger, D. 7th ed. Burlington (MA): Academic Press; 2007.

[11] Ng, EW, Geller M. A Table of Integrals of the Error Functions. J. Research NBS. 1969;73B:120.

[12] Risken H. The Fokker-Planck Equation. Methods of Solution and Applications. 2nd ed. Berlin: Springer; 1989.

[13] Erdélyi A, Magnus W, Oberhettinger F, Tricomi FG. Higher Transcendental Functions. Vol. 1. New York (NY): McGraw-Hill; 1953. 
Table 1: Inverse transforms of Laplace transforms that contain products of two parabolic cylinder functions.*

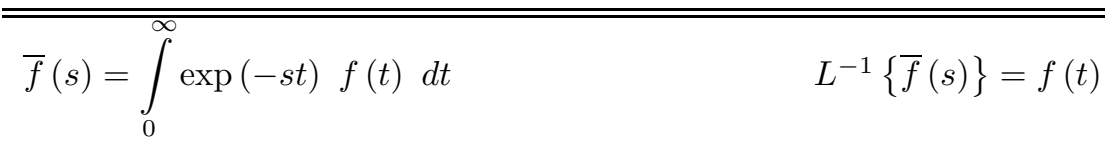

1. $\Gamma\left(\frac{s+c}{\beta}\right) D_{-(s+c) / \beta}(x) D_{-(s+c) / \beta}(y)$

$$
\begin{aligned}
& \frac{\beta \exp (-c t)}{\sqrt{1-\exp (-2 \beta t)}} \exp \left(\frac{y^{2}-x^{2}}{4}\right) \\
& \quad \times \exp \left(-\frac{(y+x \exp (-\beta t))^{2}}{2(1-\exp (-2 \beta t))}\right)
\end{aligned}
$$

2. $\Gamma\left(\frac{s+c}{\beta}\right) D_{-(s+c) / \beta}(x) D_{-1-(s+c) / \beta}(y)$

$$
\frac{\beta \sqrt{\pi} \exp (-c t)}{\sqrt{2}} \exp \left(\frac{y^{2}-x^{2}}{4}\right) \operatorname{erfc}\left(\frac{y+x \exp (-\beta t)}{\sqrt{2(1-\exp (-2 \beta t))}}\right)
$$

3. $s \Gamma\left(\frac{s+c}{\beta}\right) D_{-(s+c) / \beta}(x) D_{-1-(s+c) / \beta}(y)$

$$
\exp \left(\frac{y^{2}-x^{2}}{4}\right)\left\{\frac{\beta^{2} \exp (-(\beta+c) t)}{(1-\exp (-2 \beta t))^{\frac{3}{2}}}\right.
$$$$
-\left.\frac{\beta \sqrt{\pi}}{\sqrt{2}}\right|_{x+y=0}
$$

$$
\times(x+y \exp (-\beta t)) \exp \left(-\frac{(y+x \exp (-\beta t))^{2}}{2(1-\exp (-2 \beta t))}\right)
$$

$$
\left.-\frac{c \beta \sqrt{\pi} \exp (-c t)}{\sqrt{2}} \operatorname{erfc}\left(\frac{y+x \exp (-\beta t)}{\sqrt{2(1-\exp (-2 \beta t))}}\right)\right\}
$$

4. $\Gamma\left(\frac{s+c}{\beta}\right) D_{-(s+c) / \beta}(x) D_{-2-(s+c) / \beta}(y)$

$$
\exp \left(\frac{y^{2}-x^{2}}{4}\right)\{\beta \sqrt{1-\exp (-2 \beta t)} \exp (-c t)
$$

$$
\times \exp \left(-\frac{(y+x \exp (-\beta t))^{2}}{2(1-\exp (-2 \beta t))}\right)
$$$$
-\frac{(x \exp (-\beta t)+y) \beta \sqrt{\pi} \exp (-c t)}{\sqrt{2}}
$$$$
\left.\times \operatorname{erfc}\left(\frac{y+x \exp (-\beta t)}{\sqrt{2(1-\exp (-2 \beta t))}}\right)\right\}
$$

5. $\frac{s+c}{\beta} \Gamma\left(\frac{s+c}{\beta}\right) D_{-(s+c) / \beta}(x) D_{-2-(s+c) / \beta}(y)$

$\exp \left(\frac{y^{2}-x^{2}}{4}\right)\left\{\frac{\beta \exp (-(2 \beta+c) t)}{\sqrt{1-\exp (-2 \beta t)}}\right.$

$$
\begin{gathered}
\times \exp \left(-\frac{(y+x \exp (-\beta t))^{2}}{2(1-\exp (-2 \beta t))}\right) \\
\left.+\frac{x \beta \sqrt{\pi} \exp (-(\beta+c) t)}{\sqrt{2}} \operatorname{erfc}\left(\frac{y+x \exp (-\beta t)}{\sqrt{2(1-\exp (-2 \beta t))}}\right)\right\}
\end{gathered}
$$

6. $\frac{s+\beta+c}{\beta} \Gamma\left(\frac{s+c}{\beta}\right) D_{-(s+c) / \beta}(x) D_{-2-(s+c) / \beta}(y)$

$$
\begin{aligned}
& \exp \left(\frac{y^{2}-x^{2}}{4}\right)\left\{\frac{\beta \exp (-c t)}{\sqrt{1-\exp (-2 \beta t)}}\right. \\
& \quad \times \exp \left(-\frac{(y+x \exp (-\beta t))^{2}}{2(1-\exp (-2 \beta t))}\right) \\
& \left.-\frac{y \beta \sqrt{\pi} \exp (-c t)}{\sqrt{2}} \operatorname{erfc}\left(\frac{y+x \exp (-\beta t)}{\sqrt{2(1-\exp (-2 \beta t))}}\right)\right\}
\end{aligned}
$$

for $\operatorname{Re} s>0, \beta>0, c \geqslant 0, x+y \geqslant 0$. 
Table 2: Integral representations for products of two parabolic cylinder functions.

1. $D_{v}(x) D_{v}(y)=\frac{\exp \left(\frac{y^{2}-x^{2}}{4}\right)}{2 \Gamma(-v)} \int_{0}^{1} \frac{1}{(1-u)^{1+\frac{v}{2}} \sqrt{u}} \exp \left(-\frac{(y+x \sqrt{1-u})^{2}}{2 u}\right) d u$

$[\operatorname{Re} v<0, x+y \geqslant 0]$

2. $D_{v}(x) D_{v-1}(y)=\frac{\sqrt{\pi} \exp \left(\frac{y^{2}-x^{2}}{4}\right)}{2^{\frac{3}{2}} \Gamma(-v)} \int_{0}^{1} \frac{1}{(1-u)^{1+\frac{v}{2}}} \operatorname{erfc}\left(\frac{y+x \sqrt{1-u}}{\sqrt{2 u}}\right) d u$

$[\operatorname{Re} v<0, x+y \geqslant 0]$

3. $D_{v}(x) D_{v-1}(y)=-\frac{\exp \left(\frac{y^{2}-x^{2}}{4}\right)}{2 v \Gamma(-v)} \int_{0}^{1} \frac{x+y \sqrt{1-u}}{(1-u)^{\frac{1+v}{2}} u^{\frac{3}{2}}} \exp \left(-\frac{(y+x \sqrt{1-u})^{2}}{2 u}\right) d u-\left.\frac{\sqrt{\pi}}{v \sqrt{2} \Gamma(-v)}\right|_{x+y=0}$

$[\operatorname{Re} v<0, x+y \geqslant 0]$

4. $D_{v}(x) D_{v-2}(y)=\frac{\exp \left(\frac{y^{2}-x^{2}}{4}\right)}{2 \Gamma(-v)} \int_{0}^{1} \frac{1}{(1-u)^{1+\frac{v}{2}}}\left\{\sqrt{u} \exp \left(-\frac{(y+x \sqrt{1-u})^{2}}{2 u}\right)\right.$

$\left.-\sqrt{\frac{\pi}{2}}(x \sqrt{1-u}+y) \operatorname{erfc}\left(\frac{y+x \sqrt{1-u}}{\sqrt{2 u}}\right)\right\} d u$

$[\operatorname{Re} v<0, x+y \geqslant 0]$

5. $D_{v}(x) D_{v-2}(y)=-\frac{\exp \left(\frac{y^{2}-x^{2}}{4}\right)}{2 v \Gamma(-v)} \int_{0}^{1} \frac{1}{(1-u)^{1+\frac{v}{2}}}\left\{\frac{1-u}{\sqrt{u}} \exp \left(-\frac{(y+x \sqrt{1-u})^{2}}{2 u}\right)\right.$

$$
\left.+x \sqrt{\frac{\pi}{2}(1-u)} \operatorname{erfc}\left(\frac{y+x \sqrt{1-u}}{\sqrt{2 u}}\right)\right\} d u
$$

$[\operatorname{Re} v<0, x+y \geqslant 0]$

6. $D_{v}(x) D_{v-2}(y)=\frac{\exp \left(\frac{y^{2}-x^{2}}{4}\right)}{2(1-v) \Gamma(-v)} \int_{0}^{1} \frac{1}{(1-u)^{1+\frac{v}{2}}}\left\{\frac{1}{\sqrt{u}} \exp \left(-\frac{(y+x \sqrt{1-u})^{2}}{2 u}\right)\right.$

$$
\left.-y \sqrt{\frac{\pi}{2}} \operatorname{erfc}\left(\frac{y+x \sqrt{1-u}}{\sqrt{2 u}}\right)\right\} d u
$$

$[\operatorname{Re} v<0, x+y \geqslant 0]$

7. $D_{v}(x) D_{v-2}(y)=\frac{\exp \left(\frac{y^{2}-x^{2}}{4}\right)}{2 v(1-v) \Gamma(-v)} \int_{0}^{1} \frac{y \sqrt{1-u}(x+y \sqrt{1-u})+v u}{(1-u)^{1+\frac{v}{2}} u^{\frac{3}{2}}} \exp \left(-\frac{(y+x \sqrt{1-u})^{2}}{2 u}\right) d u$

$$
-\left.\frac{y \sqrt{\pi}}{v(v-1) \sqrt{2} \Gamma(-v)}\right|_{x+y=0}
$$

$[\operatorname{Re} v<0, x+y \geqslant 0]$

8. $D_{v}(x) D_{v+1}(y)=\frac{\exp \left(\frac{y^{2}-x^{2}}{4}\right)}{2 \Gamma(-v)} \int_{0}^{1} \frac{y u+\sqrt{1-u}(x+y \sqrt{1-u})}{(1-u)^{1+\frac{v}{2}} u^{\frac{3}{2}}} \exp \left(-\frac{(y+x \sqrt{1-u})^{2}}{2 u}\right) d u+\left.\frac{\sqrt{\pi}}{\sqrt{2} \Gamma(-v)}\right|_{x+y=0}$

$[\operatorname{Re} v<0, x+y \geqslant 0]$ 
Table 3: Limits that contain gamma functions and (products of two) parabolic cylinder functions.

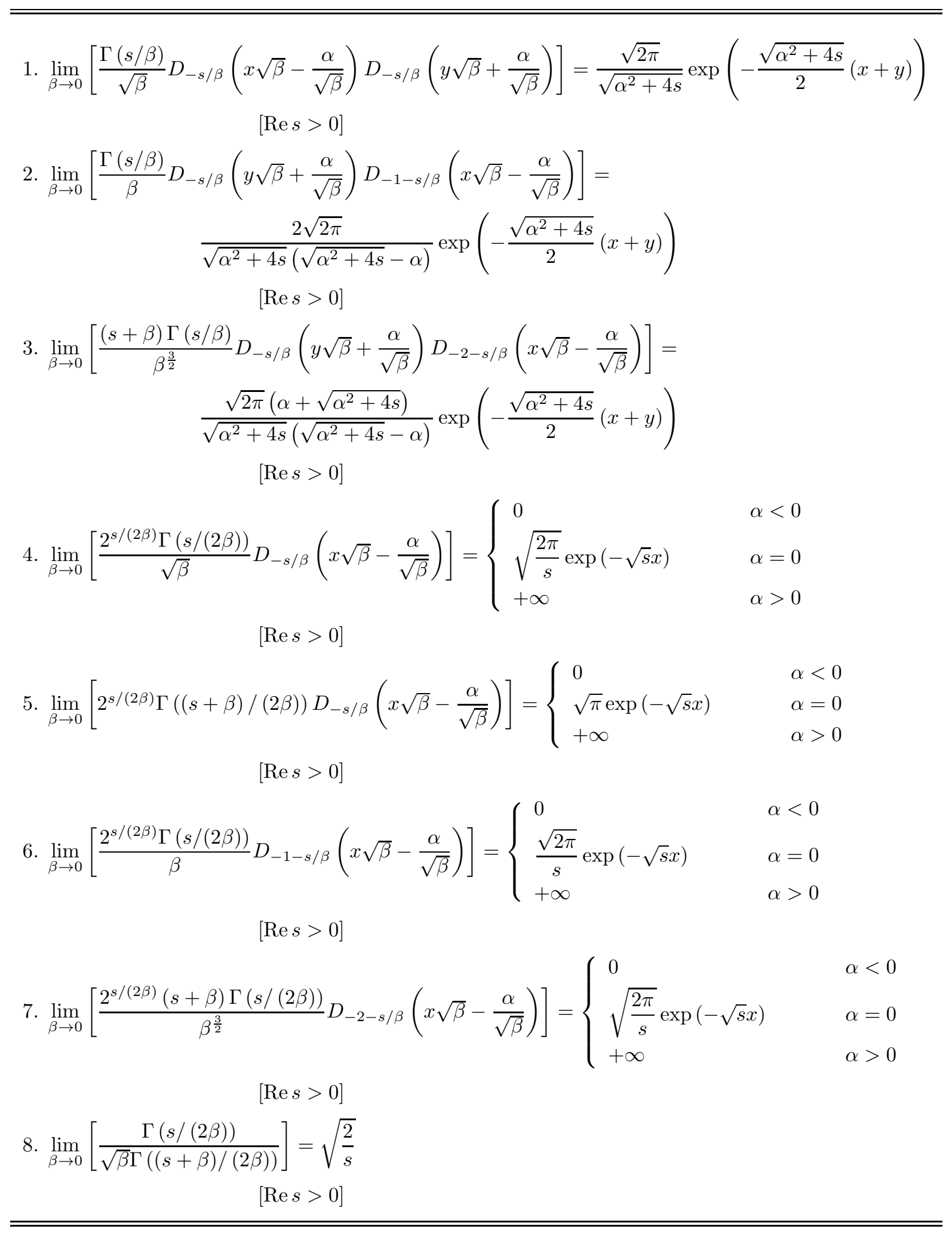

